\title{
A global analysis of molecular markers and phenotypic traits in local chicken breeds in Taiwan
}

\author{
C. S. Chang ${ }^{*}{ }^{\dagger}$, C. F. Chen*, C. Berthouly-Salazar ${ }^{\ddagger}$, O. Chazara ${ }^{\S}$, Y. P. Lee ${ }^{*}$, C. M. Chang ${ }^{\pi}$, \\ K. H. Chang*, B. Bed'Hom ${ }^{\dagger}$ and M. Tixier-Boichard ${ }^{\dagger}$ \\ * Department of Animal Science, National Chung-Hsing University, Taichung 402, Taiwan. ${ }^{\dagger}$ INRA, AgroParisTech, UMR1313 Génétique \\ Animale et Biologie Intégrative, Jouy-en-Josas 78352, France. ${ }^{\ddagger}$ Department of Zoology and Entomology, Centre for Invasion Biology, \\ University of Pretoria, Pretoria 0002, South Africa. ${ }^{5}$ Department of Pathology, University of Cambridge, Tennis Court Road, Cambridge \\ CB2 1QP, UK. "Research Center for Emerging Viral Infections and Department of Medical Biotechnology and Laboratory Science, Chang \\ Gung University, Tao-Yuan 333, Taiwan.
}

\section{Summary}

\begin{abstract}
Molecular and phenotypic data have been combined to characterize the genetic diversity of six local chicken breeds maintained with a long-term conservation programme. Hua-Tung, Hsin-Yi, Ju-Chi and Quemoy originated from Taiwan, Shek-Ki is from South China, and Nagoya is from Japan. Molecular tools included 24 microsatellite markers, melanocortin 1 receptor (alpha melanocyte stimulating hormone receptor) (MC1R), the LEIO258 marker located within the major histocompatibility complex (MHC), and mitochondrial DNA. Performance was recorded on the same individuals for body weight, panting rate in summer and antibody response (antigens: Newcastle disease virus and sheep red blood cells). A multivariate method previously proposed for taxonomy was used to combine the different data sets. Melanocortin 1 receptor (alpha melanocyte stimulating hormone receptor) and the MCW330 marker contributed the most to the first axis of the multiple coinertia analysis of molecular markers. Melanocortin 1 receptor (alpha melanocyte stimulating hormone receptor) showed evidence of selection, probably related to its effect on feather colour. The MHC exhibited a large diversity, with 16 alleles of the LEIO258 marker. Immune response traits contributed the most to the principal component analysis of phenotypic data. Eight mitochondrial DNA haplotypes related to clades A, B, C and E were distributed across breeds and revealed an important contribution of Indian and European breeds to Ju-Chi, Quemoy and Hsin-Yi. Phenotypic data contributed less than molecular data to the combined analysis, and two markers, LEIO258 and LEIO228, contributed the most. The combined analysis could clearly discriminate all breeds, except JuChi, which was similar to Quemoy for many criteria, except immune response.
\end{abstract}

Keywords local chicken, microsatellites, melanocortin 1 receptor (alpha melanocyte stimulating hormone receptor), major histocompatibility complex, mitochondrial DNA, immune response, body weight.

\section{Introduction}

Preserving local breeds is an important issue for the conservation of domestic animal genetic diversity (FAO 2007). There is a wide array of chicken breeds with abundant phenotypic diversity, as a result of domestication and selection. Commercial lines and industrialized livestock

Address for correspondence

C. S. Chang, Department of Animal Science, National Chung-Hsing University, Taichung 402, Taiwan.

E-mail: fattelos@gmail.com

Accepted for publication 12 May 2011 production systems have spread over all continents. Local breeds have low productivity but may harbour specific features as a result of adaptation to their environment. A subtropical island such as Taiwan $\left(21^{\circ} 90^{\prime}-25^{\circ} 29^{\prime} \mathrm{N}\right.$; $\left.120^{\circ} 05^{\prime}-122^{\circ} 00^{\prime} \mathrm{E}\right)$ provides an interesting framework to study genetic diversity of local chicken breeds, because of its climate and its geographic situation. Initially, native chickens were raised in Taiwan by aboriginal tribes. Historical records (Lee 2006) indicate several waves of introduction of domestic chickens from China (400 years ago), Europe (300 years ago) and Japan (100 years ago). Chicken populations were then exposed to the subtropical climate, with some selection by local farmers. 
A conservation programme was set up in 1982 for four local chicken breeds and two imported breeds at National Chung-Hsing University (NCHU) in Taiwan. These breeds were characterized for an array of traits, such as disease resistance, meat quality (Lee 2006), and molecular diversity (Chen et al. 2004 \& Berthouly et al. 2008). However, the latter study mainly addressed neutral diversity, which is not sufficient to assess the global conservation potential of local breeds.

The aim of this study was to extend the diversity study to functional traits and to enhance current knowledge of breed history by sequencing mitochondrial DNA (mtDNA). We added two hypervariable microsatellite markers (LEIO228 and LEIO192) and two markers associated with functional information: (i) the MC1R gene, which is one of the main loci controlling feather colour (Takeuchi et al. 1996; Kerje et al. 2003) and (ii) the LEIO258 marker located within the MHC locus, involved in response to infectious diseases (Lamont 1989). Because individual phenotypes were also available for an array of traits, a methodological approach is proposed to combine molecular (discrete) and phenotypic (continuous) data, in order to provide a comprehensive view of the genetic diversity of these breeds.

\section{Materials and methods}

\section{Experimental chickens}

Six local chicken breeds have been maintained in a conservation programme at NCHU since 1982 (Lee 2006; Fig. 1). Hsin-Yi shows red feathers, white skin and blue shanks.
Ju-Chi and Hua-Tung have black plumage and black shanks. Quemoy has black plumage with gold-laced feathers on the neck, white skin, and blue shanks. Shek-Ki has yellow skin, yellow feathers and yellow shanks (the 'triple-yellow'). Nagoya has yellow plumage with black tail and blue shanks.

The management of experimental chickens followed a routine procedure (Chao \& Lee 2001). A representative set of 50 animals, 25 males and 25 females, was sampled according to pedigree records. Blood was collected for DNA extraction at 40 weeks of age, and all animals sampled had individual performance records. Birds were produced in four successive hatches for the six breeds, and breeds were reared together.

\section{Molecular markers}

\section{Microsatellite markers}

A set of 24 microsatellite markers from the AvianDiv European project (Hillel et al. 2003; Table S1) was used to analyse within- and between-breed diversity. It included the 22 markers described previously by Berthouly et al. (2008) and two additional markers, LEIO228 and LEIO192, which have numerous alleles (14 for each marker). Markers were genotyped with the protocol described in Berthouly et al. (2008).

\section{Mitochondrial DNA}

The hypervariable sequence 1 (HVS-I) of the D-loop region was amplified using the same primers (Table S2) following the protocol of Liu et al. (2006).

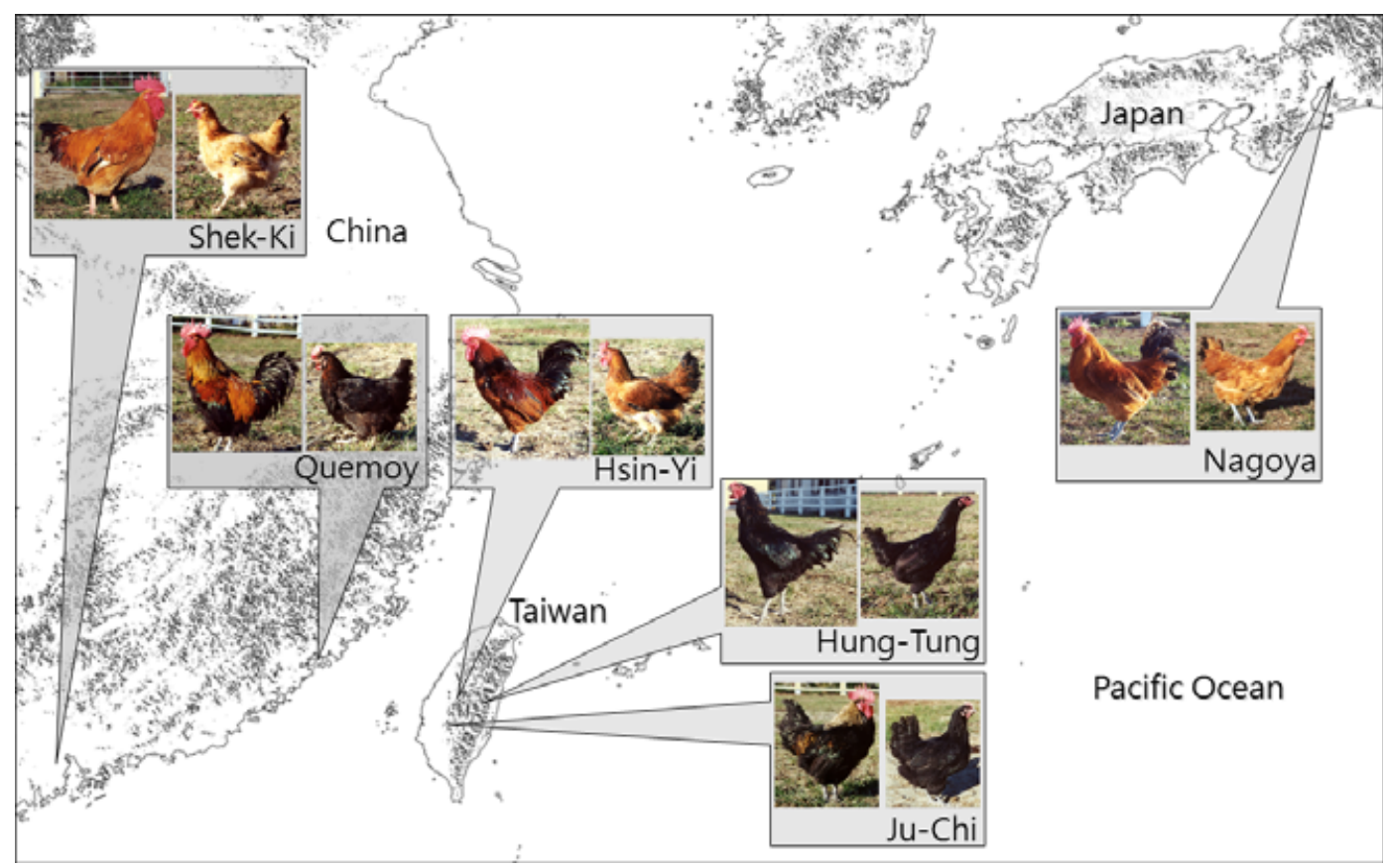

Figure 1 The six local chicken breeds under conservation at the National Chung-Hsing University with their region of origin. Hua-Tung (HT), Hsin-Yi (HY), Ju-Chi (JC) and Quemoy (QM) are from the Taiwan region, Shek-Ki (SK) is from Hong-Kong, and Nagoya (NG) is from Japan. 
Melanocortin 1 receptor (alpha melanocyte stimulating hormone receptor)

The chicken MC1R gene-coding sequence was amplified with PCR primers (Table S2) and protocol as described by Chen et al. (2005).

\section{LEIO258 marker}

The LEIO258 marker is an atypical Variable Number of Tandem Repeat marker that exhibits a very good correlation with serological typing of the chicken MHC (Fulton et al. 2006). PCR primers (Table S2) and protocol are the same as those used by Fulton et al. (2006).

\section{Performance traits}

Body weight was measured every two weeks from hatch to 18 weeks of age, and again at 47 weeks of age. Three measures were kept for the analysis: initial body weight at hatch (BWO), body weight at the time of rapid growth (BW12), and mature body weight (BW47). Panting is generally observed when the ambient temperature is above $29{ }^{\circ} \mathrm{C}$ with $50 \%$ humidity (Weaver 2002). Panting was recorded individually on a daily basis between 24 and 27 weeks of age, in the afternoon of the hot season when the ambient temperature was higher than $30{ }^{\circ} \mathrm{C}$ (highest $36{ }^{\circ} \mathrm{C}$, lowest $31{ }^{\circ} \mathrm{C}$ and average $33.5{ }^{\circ} \mathrm{C}$ with $61 \%$ humidity). Individual panting rate was calculated as the ratio of number of days where panting occurred divided by the total number of observed days.

Immune response was investigated using two different antigens. Sheep red blood cells (SRBC) are frequently used as a non-pathogenic antigen for evaluating antibody response in selection experiments, and antibody response to Newcastle disease virus (NDV) vaccination can be used as a selection criterion for immune response (Lamont et al. 2003). Newcastle disease is a major poultry disease in Taiwan (Yang et al. 1999), and vaccination against NDV is a standard procedure recommended by government sanitary authorities. All day-old chicks were vaccinated against NDV in this study. At 8 weeks of age, all chickens were vaccinated again with $0.5-\mathrm{ml} \mathrm{NDV}$ vaccine, and serum was collected 7 and 14 days later. Haemagglutination inhibition tests were used to determine antibody titres against NDV. At 11 and 13 weeks of age, chickens were injected with $0.1 \mathrm{ml}$ $0.25 \%$ SRBC intravenously, and serum was collected 7 days later. Antibody titres of chickens against SRBC were determined by haemagglutination tests. The antibody titre was expressed as the $\log _{2}$ of the reciprocal of the highest dilution.

The kinetics of antibody titres was studied by calculating NDD as the difference between anti-ND antibody titre at day 14 and anti-ND antibody titre at day 7 post-inoculation, and SRBCD was calculated as the difference between anti-
SRBC antibody titre at secondary response and anti-SRBC antibody titre at primary response.

\section{Sequencing and sequence analysis}

PCR products of mtDNA, MC1R and LEIO258 were sequenced by Eurofins MWG Operon Company. Sequence alignment, editing and processing were performed with BIOEDIT 7.0.9.0 software (Hall 1999) and the STADEN package (Staden et al. 1998). Mitochondrial DNA and LEIO258 sequence alignment and polymorphism identification were carried out using BIOEDIT. Haplotypes were scored manually. The median-joining networks (Bandelt et al. 1999) were constructed for mitochondrial DNA data using the program Network 4.516 (http://www.fluxus-engineering.com/ sharenet_rn.htm). The length variants (number of repeats) and polymorphic sites (either single-nucleotide polymorphisms (SNP) or deletions) of LEIO258 were checked for each allele. MC1R SNP analysis was carried out with the STADEN package (Staden et al. 1998) in order to define genotypes and haplotypes.

\section{Analysis of genotypes}

Allele frequencies, number of alleles and expected and observed heterozygosity were calculated using GENETIX 4.04 (Belkhir et al. 2004). Genetic distances (Nei et al. 1983) were calculated, and neighbour-joining (NJ) trees were established using POPULATIONS 1.2.30 (Langella 1999).

All marker data, including microsatellites, MC1R, and LEIO258, were merged and treated with a Multiple CO-inertia Analysis (MCOA) using R (R Core Development Team 2006) with the ADE-4 package (Chessel et al. 2004; Dray \& Dufour 2007). This method makes the extraction of common information from separate analyses possible, by setting up a reference typology and comparing each typology separately. The efficiency of a marker is assessed by its typological value $\left(T_{\mathrm{V}}\right)$, which is the contribution of the marker to the construction of the reference typology, which is equal to the product of the variance (Var) multiplied by the congruence with the consensus $\operatorname{Cos}^{2}$ (i.e. the correlation between the scores of individual locus tables and the synthetic variable of the same rank) (Laloë et al. 2007).

\section{Analysis of performance data}

Body weight, antibody titres and panting rate were analysed using the general linear model (GLM) procedure of sAs software (SAS Institute 2008). Breed and sex were treated as fixed effects with a breed $\times$ sex interaction. Partial correlations between body weight and panting rate were calculated for each sex after accounting for the breed effect. Principal 
component analysis (PCA) was performed with the ADE-4 package from $\mathrm{R}$, in order to identify the best combination of variables that explained most of the variance between breeds.

\section{Combining molecular and phenotypic data}

The Hill \& Smith (1976) method was used to combine discrete and continuous variables to compare populations. This method is a combination of an internal correspondence analysis for discrete data, i.e. the molecular marker data (Laloë et al. 2002), and a PCA for continuous variables, i.e. performance traits. It was implemented with functions of the ADE-4 package from the $\mathrm{R}$ software.

\section{Results}

\section{Genetic analyses of molecular markers}

Ranking of breeds for heterozygosity values was similar to previous results of Berthouly et al. (2008), with a maximum value of 0.598 for Hua-Tung (instead of 0.584) and a minimum value of 0.412 for Shek-Ki (instead of 0.423). Hua-Tung and Ju-Chi exhibited weakly negative values for $F_{\text {IS }}(-0.028$ and -0.048 , respectively), suggesting a slight excess of heterozygotes, whereas Shek-Ki exhibited the highest $F_{\text {IS }}$ (0.079 as compared to 0.068 with 22 markers), which differed significantly from zero and indicated some inbreeding.

The mean Fst values ranged from 0.22 (Hua-Tung) to 0.35 (Nagoya). Shek-Ki showed the second highest mean Fst value (0.31), and mean Fst values varied from 0.24 to 0.27 for the other Taiwanese breeds (Table S3).
Melanocortin 1 receptor (alpha melanocyte stimulating hormone receptor)

There were six SNPs that defined five haplotypes, corresponding to four different protein sequences, which combined in eight genotypes (Tables 1 and S4).

Hsin-Yi and Shek-Ki shared haplotype 3 with a high frequency and were the only breeds to carry haplotype 5 at a low frequency, and they clustered together in the NJ tree (Fig. S1A). Haplotype 4 was almost fixed in Nagoya and was also found in Shek-Ki and Hsin-Yi, but at quite different frequencies, explaining the clustering of these three breeds in the NJ tree. Ju-Chi, Hua-Tung and Quemoy exhibited a high frequency of haplotype 1; Hua-Tung was the only breed to carry haplotype 2. Consequently, Ju-Chi and Quemoy grouped in the same cluster, and Hua-Tung had a basal position in the tree.

Shek-Ki was the only breed to exhibit a high heterozygosity for MC1R (0.578; Table S4) and a strongly negative $F_{\text {IS }}(-0.313$; data not shown).

\section{LEIO258}

A total of 16 alleles were found in the six breeds; six of them had never been described and were breed specific (Table 2). Hua-Tung had the highest number of alleles and the highest heterozygosity value; four alleles out of seven were private. The four alleles found in Shek-Ki were all private. The lowest heterozygosity value was found for Ju-Chi, whereas the highest $F_{\text {IS }}$ value was observed for Hsin-Yi.

Ju-Chi, Quemoy and Hsin-Yi shared allele 309 at a rather high frequency and grouped in the same cluster of the NJ

Table $1 M C 1 R$ haplotypes, number of haplotypes and percentage in each breed.

\begin{tabular}{|c|c|c|c|c|c|c|c|c|c|c|c|c|}
\hline \multirow[b]{5}{*}{ Haplotypes } & \multicolumn{6}{|c|}{ SNP position in the coding sequence } & \multicolumn{6}{|c|}{ Number of haplotypes (percentage) per breed } \\
\hline & 212 & 274 & 427 & 636 & 637 & 644 & & & & & & \\
\hline & $\mathrm{T}>\mathrm{A}$ & $G>A$ & $A>G$ & $G>A$ & $\mathrm{~T}>\mathrm{C}$ & $A>C$ & & & & & & \\
\hline & \multicolumn{12}{|c|}{ Amino acid position } \\
\hline & 71 & 92 & 143 & 212 & 213 & 215 & $H \sin -Y i$ & Ju-Chi & Hua-Tung & Quemoy & Shek-Ki & Nagoya \\
\hline \multirow[t]{2}{*}{1} & $\mathrm{C}$ & A & A & G & $\mathrm{T}$ & A & $1(1 \%)$ & $88(96 \%)$ & $77(84 \%)$ & $91(99 \%)$ & & \\
\hline & Thr & Lys & Thr & Ala & Cys & His & & & & & & \\
\hline \multirow[t]{2}{*}{2} & $\mathrm{C}$ & A & A & A & $\mathrm{T}$ & A & & & $8(9 \%)$ & & & \\
\hline & Thr & Lys & Thr & Ala & Cys & His & & & & & & \\
\hline \multirow[t]{2}{*}{3} & $\mathrm{C}$ & A & A & $G$ & $\mathrm{~T}$ & $C$ & $85(92 \%)$ & $4(4 \%)$ & $7(7 \%)$ & $1(1 \%)$ & $62(69 \%)$ & $1(1 \%)$ \\
\hline & Thr & Lys & Thr & Ala & Cys & Pro & & & & & & \\
\hline \multirow[t]{2}{*}{4} & $\mathrm{~T}$ & $G$ & G & A & $\mathrm{C}$ & $A$ & $5(5 \%)$ & & & & $27(30 \%)$ & $93(99 \%)$ \\
\hline & Met & Glu & Ala & Ala & Arg & His & & & & & & \\
\hline \multirow[t]{2}{*}{5 (wild type) } & $\mathrm{T}$ & G & $A$ & $G$ & T & A & $2(2 \%)$ & & & & $1(1 \%)$ & \\
\hline & Met & Glu & Thr & Ala & Cys & His & & & & & & \\
\hline
\end{tabular}

$M C 1 R$, melanocortin 1 receptor (alpha melanocyte stimulating hormone receptor). 
Table 2 Allelic frequencies of the LEIO258 marker for the six local chicken breeds.

\begin{tabular}{|c|c|c|c|c|c|c|c|}
\hline \multicolumn{7}{|c|}{ Allele frequency } & \multirow{3}{*}{$\begin{array}{l}\text { Corresponding } \\
\text { B haplotype }^{1}\end{array}$} \\
\hline Allele & Hisn-Yi & Ju-Chi & Hua-Tung & Quemoy & Shek-Ki & Nagoya & \\
\hline N & 47 & 48 & 48 & 48 & 48 & 48 & \\
\hline $181^{2}$ & & & & & & 0.40 & NA \\
\hline 182 & & & & & 0.21 & & 4 \\
\hline 193 & 0.11 & & 0.06 & & & & $11,15.1,27,61$ \\
\hline 205 & & 0.17 & & 0.20 & & & 13 \\
\hline $217^{2}$ & 0.02 & & & & & & NA \\
\hline $241^{2}$ & & & & & 0.14 & & NA \\
\hline 249 & & 0.05 & 0.19 & 0.01 & & 0.56 & $15.2,22,73$ \\
\hline $273^{2}$ & & & & & 0.34 & & NA \\
\hline 295 & & & 0.22 & & & & 5 \\
\hline 309 & 0.44 & 0.77 & 0.07 & 0.54 & & 0.04 & $10,24,26,76$ \\
\hline $319^{2}$ & & & 0.11 & & & & NA \\
\hline 345 & & & & & 0.31 & & 14 \\
\hline 357 & & & 0.16 & & & & $5.1,6.1,21,75$ \\
\hline 381 & & 0.01 & & 0.25 & & & 13.1 \\
\hline $419^{2}$ & & & 0.19 & & & & NA \\
\hline 443 & 0.44 & & & & & & 6 \\
\hline$H_{e}$ & 0.61 & 0.38 & 0.84 & 0.61 & 0.73 & 0.53 & \\
\hline$H_{o}$ & 0.51 & 0.40 & 0.83 & 0.65 & 0.71 & 0.58 & \\
\hline$F_{\mathrm{IS}}$ & 0.17 & -0.04 & 0.01 & -0.06 & 0.03 & -0.10 & \\
\hline
\end{tabular}

${ }^{1}$ Corresponding B haplotypes with Fulton et al. (2006).

${ }^{2}$ Newly described private alleles.

tree. Nagoya and, to a lesser extent, Hua-Tung, showed a high frequency of allele 249 and clustered together on the NJ tree; allele 295 (private) was the most frequent in the Hua-Tung breed, which might contribute to the long branch length of this breed in the NJ tree. Shek-Ki was the most different, because it did not share any allele with the other breeds, and it represents an independent branch of the NJ tree (Fig. S1B).

\section{Multivariate analysis}

The first two axes of MCOA analysis with the 24 microsatellite markers explained $51.1 \%$ and $21.2 \%$ of the observed inertia, respectively. The two markers MCW330 and MCW034 contributed the most to the construction of the first axis, with $8.6 \%$ and $8.3 \%$, respectively. Results from MCOA analysis after addition of MC1R and LEIO258 data to the microsatellite data are shown in Fig. 2. The first two axes, of MCOA analysis explained $50.4 \%$ and $24 \%$ of the observed inertia, respectively. The two markers MC1R and MCW330 contributed the most to the construction of the first axis, with $10 \%$ and $6.7 \%$, respectively. LEIO258 ranked 7 th of 26 markers, with a contribution of $5.2 \%$. The first axis separated Nagoya from the set of Taiwanese-Chinese breeds. Hsin-Yi, Hua-Tung and Ju-Chi were strongly overlapping each other. The two markers MC1R and LEIO228 contributed the most to the construction of the second axis, with $22.5 \%$ and $13.8 \%$, respectively. LEIO258 ranked 5th with a contribution of $8.1 \%$. The second axis separated Quemoy and Shek-Ki.

\section{Phenotypic data}

Breeds differed significantly for body weight at all stages (Table 3). There was a significant breed $\times$ sex interaction $(P<0.05)$ for mature body weight, which was highest in Hua-Tung and Nagoya for males and in Nagoya and ShekKi for females. Ju-Chi and Quemoy exhibited the lowest body weight for both sexes, and Hsin-Yi showed intermediate values. There was a significant $(P<0.01)$ breed $\times$ sex interaction for panting rate. Hsin-Yi, Shek-Ki and Hua-Tung showed higher panting rates in males, whereas Shek-Ki and Nagoya showed the highest values in females. Quemoy exhibited a low value in males, but not in females. Ju-Chi exhibited a low panting rate for both sexes. On average, females had a higher panting rate and lower body weight than males. Partial correlation between BW47 and panting rate was moderate and positive ( 0.30 in females, 0.23 in males, $P<0.01)$.

Results for immunological traits are listed in Table 4. Breeds differed both in kinetics and in final titres. Quemoy exhibited a faster antibody response for both ND and SRBC, and final titres were highest in Quemoy for ND antibodies, but not for SRBC antibodies. Ju-Chi had the lowest values for ND response. A negative correlation was observed between initial values (ND7 or SRBC1) and the subsequent 
Figure 2 Position of six local breeds obtained by multiple coinertia analysis with 24 microsatellite markers, MC1R and LEIO258. HT: Hua-Tung, HY: Hsin-Yi, JC: Ju-Chi, QM: Quemoy, SK: Shek-Ki and NG: Nagoya.

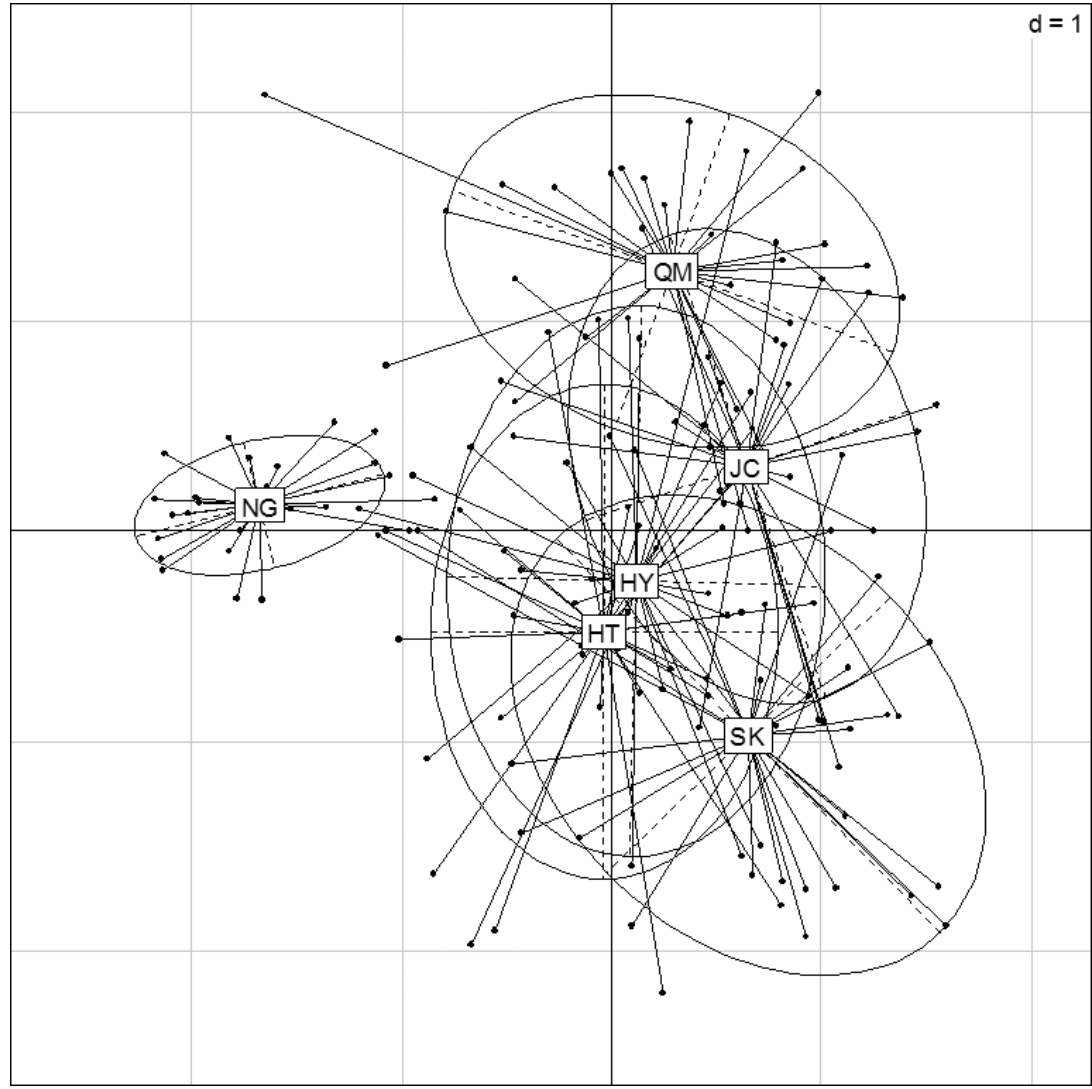

Table 3 Least squares means of the phenotypic traits for the six local breeds.

\begin{tabular}{|c|c|c|c|c|c|c|}
\hline Traits $^{1}$ & $H \sin -Y i$ & Ju-Chi & Hua-Tung & Quemoy & Shek-Ki & Nagoya \\
\hline \multicolumn{7}{|l|}{ Males } \\
\hline BWO & $36.2 \pm 0.7^{\mathrm{ab}}$ & $34.8 \pm 0.7^{b c}$ & $36.7 \pm 0.6^{a}$ & $33.2 \pm 0.6^{c}$ & $35.8 \pm 0.8^{\mathrm{ab}}$ & $34.5 \pm 0.6^{b c}$ \\
\hline BW12 & $1281 \pm 35^{b}$ & $1192 \pm 36^{c}$ & $1310 \pm 34^{b}$ & $1093 \pm 34^{c}$ & $1545 \pm 40^{\mathrm{a}}$ & $1240 \pm 33^{b}$ \\
\hline BW47 & $2687 \pm 65^{c}$ & $2476 \pm 67^{d}$ & $3155 \pm 64^{a}$ & $2361 \pm 64^{d}$ & $2957 \pm 75^{\mathrm{b}}$ & $3112 \pm 62^{\mathrm{ab}}$ \\
\hline PANT & $29 \pm 4^{a}$ & $13 \pm 4^{\mathrm{bcd}}$ & $23 \pm 4^{\mathrm{ab}}$ & $13 \pm 4^{\mathrm{bcd}}$ & $28 \pm 4^{\mathrm{ab}}$ & $18 \pm 3^{b c}$ \\
\hline \multicolumn{7}{|l|}{ Females } \\
\hline BWO & $36.5 \pm 0.7^{\mathrm{a}}$ & $33.9 \pm 0.7^{b c}$ & $36.2 \pm 0.6^{a}$ & $32.7 \pm 0.6^{c}$ & $34.6 \pm 0.7^{\mathrm{ab}}$ & $35.5 \pm 0.6^{\mathrm{ab}}$ \\
\hline BW12 & $1032 \pm 34^{\mathrm{a}}$ & $936 \pm 32^{b c}$ & $1049 \pm 31^{a}$ & $896 \pm 31^{c}$ & $1170 \pm 33^{a}$ & $1028 \pm 30^{\mathrm{ab}}$ \\
\hline BW47 & $2013 \pm 75^{\mathrm{b}}$ & $1492 \pm 71^{c}$ & $2022 \pm 70^{b}$ & $1522 \pm 70^{c}$ & $2197 \pm 73^{\mathrm{ab}}$ & $2257 \pm 68^{\mathrm{a}}$ \\
\hline PANT & $56 \pm 5^{\mathrm{bc}}$ & $39 \pm 5^{d}$ & $51 \pm 5^{\mathrm{bcd}}$ & $60 \pm 5^{\mathrm{bc}}$ & $76 \pm 5^{a}$ & $62 \pm 5^{\mathrm{ab}}$ \\
\hline
\end{tabular}

${ }^{1}$ BWO, body weight (grams) at 0 day of age; BW12, body weight (grams) at 12 weeks of age; BW47, body weight (grams) at 47 weeks of age; PANT, panting rate.

${ }^{\mathrm{a}, \mathrm{b}}$ Means $\pm \mathrm{SE}$ within a row with no common superscript differ significantly $(P<0.05)$.

Table 4 Least squares means of the immunological traits for the six local breeds.

\begin{tabular}{|c|c|c|c|c|c|c|}
\hline Trait $^{1}$ & $H \sin -Y i$ & Ju-Chi & Hua-Tung & Quemoy & Shek-Ki & Nagoya \\
\hline ND7 & $8.8 \pm 0.3^{a}$ & $7.4 \pm 0.3^{b}$ & $7.9 \pm 0.3^{b}$ & $8.7 \pm 0.3^{a}$ & $7.5 \pm 0.3^{b}$ & $8.1 \pm 0.3^{\mathrm{ab}}$ \\
\hline NDD & $1.3 \pm 0.3^{c}$ & $2.3 \pm 0.3^{\mathrm{ab}}$ & $2 \pm 0.3^{b c}$ & $2.5 \pm 0.3^{a b}$ & $3.1 \pm 0.4^{\mathrm{a}}$ & $2.1 \pm 0.3^{b c}$ \\
\hline SRBC1 & $4.1 \pm 0.3^{\mathrm{ab}}$ & $2.8 \pm 0.3^{c}$ & $3.5 \pm 0.3^{b c}$ & $4.6 \pm 0.3^{a}$ & $4.3 \pm 0.3^{\mathrm{ab}}$ & $3.6 \pm 0.3^{b c}$ \\
\hline SRBCD & $0.9 \pm 0.3^{\mathrm{abc}}$ & $1.7 \pm 0.3^{\mathrm{a}}$ & $0.8 \pm 0.3^{b c}$ & $0.2 \pm 0.3^{c}$ & $0 \pm 0.3^{c}$ & $1.5 \pm 0.3^{\mathrm{ab}}$ \\
\hline
\end{tabular}

${ }^{\mathrm{a}, \mathrm{b}}$ Means $\pm \mathrm{SE}$ within a row with no common superscript differ significantly $(P<0.05)$

${ }^{1} \mathrm{ND} 7$, anti-ND antibody titre at day 7 post-inoculation; NDD, difference between anti-ND antibody titre at day 14 and anti-ND antibody titre at day 7 post-inoculation; SRBC1, anti-SRBC antibody titre at primary response; SRBCD, anti-SRBC antibody titre at secondary response minus anti-SRBC antibody titre at primary response. 


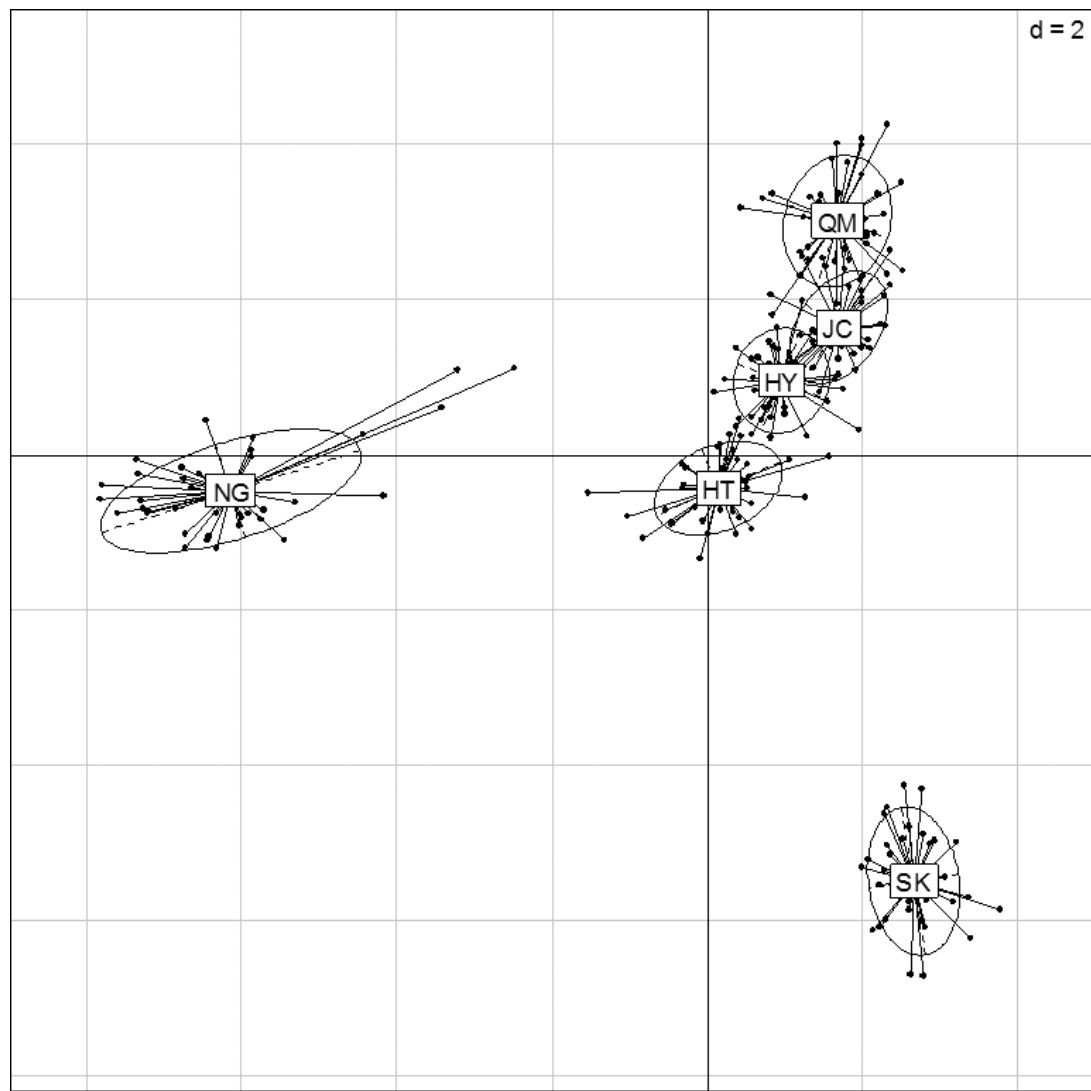

Figure 3 Position of six local breeds obtained by the Hill \& Smith method combining molecular and phenotypic data. HT: Hua-Tung, HY: Hsin-Yi, JC: Ju-Chi, QM: Quemoy, SK: Shek-Ki and NG: Nagoya. change in antibody titres $(r=-0.58$ between ND7 and NDD; $r=-0.86$ between SRBC1 and SRBCD). Correlations between body weight and immune response were not significant (data not shown).

The first two axes of PCA analysis explained 23.4\% and $20.5 \%$ of the observed inertia, respectively. The two factors ND7 and SRBCD contributed the most to the construction of the first axis, with $27.6 \%$ and SRBCD $24.1 \%$, respectively; factors contributing most to the second axis were NDD and BW47, with 23.9\% and 19.1\%, respectively. Projection of individual data on the two axes showed a total overlap between breeds which could not be discriminated (data not shown).

Hill \& Smith method for combining molecular and phenotypic data

Figure 3 shows the distribution of the six breeds using the Hill \& Smith method on mixed data combining molecular and phenotypic data. The first two axes explained 9.9\% and $7.2 \%$ of the observed inertia, respectively. The two markers LEIO228 and LEIO258 contributed the most to the construction of the first axis, with $12.6 \%$ and $9.3 \%$, respectively. Melanocortin 1 receptor (alpha melanocyte stimulating hormone receptor) ranked $13 \mathrm{th}$, and the first phenotypic factors contributing to this axis were SRBCD and SRBC1, which ranked 27 and 28th. The discriminant analysis showed that each breed made one group, except Ju-Chi and Quemoy.

\section{Mitochondrial DNA phylogeny}

There were 19 substitutions in a 497-bp mtDNA HVS-I region, and all substitutions were transitions. The eight haplotypes could be matched to previously published sequences, and the same codification as Liu et al. (2006) was used for further analysis and comparison.

Four clades were observed: A, B, C and E (Table 5). Three haplotypes were observed for clades A and E, while clades B and $\mathrm{C}$ were only represented by one haplotype each. HsinYi, Ju-Chi and Quemoy were distributed in clades A and E sharing the same haplotypes. Haplotype E1 was very frequent in the Quemoy breed, followed by the Hsin-Yi breed. Nagoya was also found in clade A and E and was the only one to exhibit the A3 haplotype. Hua-Tung was in clade A and $\mathrm{C}$, and Shek-Ki was in clade B and C. The medianjoining network showed that clades $\mathrm{A}$ and $\mathrm{E}$ were the dominant haplogroups in Taiwan local breeds, whereas clades $\mathrm{B}$ and $\mathrm{C}$ were minor haplogroups. Among the eight haplotypes, three were breed specific and exhibited moderate to high frequencies: A2 in Hua-Tung, A3 in Nagoya and B1 in Shek-Ki. 
Table 5 Identification and frequency of mitochondrial DNA haplotypes for the six breeds.

\begin{tabular}{|c|c|c|c|c|c|c|c|c|}
\hline Haplotype $^{1}$ & $H \sin -Y i$ & Ju-Chi & Hua-Tung & Quemoy & Shek-Ki & Nagoya & Total & $\begin{array}{l}\text { Genbank } \\
\text { accession } \\
\text { number }\end{array}$ \\
\hline A1 & $17(35 \%)$ & $34(71 \%)$ & $11(23 \%)$ & $1(2 \%)$ & & $25(54 \%)$ & $88(31 \%)$ & HQ022887 \\
\hline A2 & & & $27(57 \%)$ & & & & $27(9 \%)$ & HQ022888 \\
\hline$A 3$ & & & & & & $17(37 \%)$ & $17(6 \%)$ & HQ022886 \\
\hline B1 & & & & & $35(73 \%)$ & & $35(12 \%)$ & HQ022881 \\
\hline $\mathrm{C} 1$ & & & $9(19 \%)$ & & $13(27 \%)$ & & $22(8 \%)$ & HQ022885 \\
\hline E1 & $10(21 \%)$ & $9(19 \%)$ & & $42(88 \%)$ & & & $61(21 \%)$ & HQ022883 \\
\hline E6 & $19(40 \%)$ & & & & & $4(9 \%)$ & $23(8 \%)$ & HQ022882 \\
\hline E9 & $2(4 \%)$ & $5(10 \%)$ & & $5(10 \%)$ & & & $12(4 \%)$ & HQ022884 \\
\hline
\end{tabular}

${ }^{1}$ Mitochondrial DNA haplotypes are named after Liu et al. (2006).

\section{Discussion}

\section{History of breeds}

The history of multiple introductions of chickens in Taiwan from China, Europe and Japan is consistent with the finding of four clades of mtDNA. The high frequency of clade E reveals an important contribution of breeds from India or Europe, which generally exhibit a high frequency of haplotypes of the E group (Liu et al. 2006; Berthouly-Salazar et al. 2010). The Quemoy exhibited the highest frequency of E haplotypes, which is consistent with the role of the Quemoy Island as an important harbour for trading between China and Taiwan, and a likely place for introduction of exotic chickens to Taiwan hundreds years ago. The Hsin-Yi was considered the popular backyard chicken in the 1970s (Lee 2006). This breed has undergone introgression from Indian or European descent, because it also exhibited a high frequency of E haplotypes. By contrast, the Ju-Chi exhibited the highest frequency of A1 haplotypes, which suggests that this breed was influenced less by introductions of western commercial breeds and could represent a more ancient breed in Taiwan. Hua-Tung, kept for cock fighting, did not carry any E haplotypes and was the only breed from Taiwan to carry the A2 and C1 haplotypes, which can be found in China and Japan. Haplotypes A1 and C1 have been found in the Shamo breed, which is also a game bird (Liu et al. 2006), and A2 was found in Hinai-dori, a Japanese local breed. Thus, the Hua-Tung is a typical Asiatic breed which has probably been crossed with other breeds, as shown by its high heterozygosity level for microsatellite markers. The Shek-Ki exhibited haplotypes from haplogroups B and C, which is consistent with its origin in South China (HongKong region), because $\mathrm{B} 1$ and $\mathrm{C} 1$ haplotypes have been previously described in Chinese breeds; B1 is found in the Qinyan breed from Guang-Dong province (Liu et al. 2006). The $\mathrm{C}$ haplogroup is generally found in Japanese breeds, but was not found in Nagoya. This result is consistent with previous observations of haplotypes A1 and A3 in Nagoyadori (Liu et al. 2006).

\section{Neutral markers vs. markers with a functional effect}

Microsatellite markers may be considered neutral, whereas MC1R and MHC alleles are related to a function or a phenotype. The multivariate analysis showed that MC1R was the most discriminative marker and exhibited the highest typological value. This can be explained by the association of MC1R with feather colour, which is a phenotypic trait that farmers could easily select for in order to obtain a breed with a uniform appearance. Indeed, haplotypes identified in the Taiwanese breeds could be matched with alleles at the Extension (E) locus: haplotypes 1 and 2 were previously associated to the extended black allele (Takeuchi et al. 1996; Kerje et al. 2003), and these haplotypes have also been found in some wild birds (Mundy 2005); haplotype 3 has been previously associated to either the buttercup allele (Kerje et al. 2003) or the brown allele (Ling et al. 2003); haplotype 4 has been previously associated to the wheaten or yellow alleles (Ling et al. 2003), and haplotype 5 is the wild-type allele. The plumage colour of breeds Ju-Chi, HuaTung and Quemoy is mostly black, which fits with their high frequency of haplotype 1. Nagoya has a yellow plumage colour, which fits with the high frequency of haplotype 4. Hsin-Yi has red plumage, which fits with the high frequency of haplotype 3; both the buttercup and the brown alleles give a mostly red plumage and differ in down colour at hatch (Smyth 1996). Shek-Ki segregates for haplotypes 3 and 4, with a higher frequency for haplotype 3 , which is consistent with its yellow plumage. Haplotype 4 is mainly present in the heterozygous state. Indeed, the yellow allele at the $E$ locus is expected to have a recessive effect on plumage colour, as compared to the buttercup allele (Smyth 1996). Thus, the MC1R gene represents a typical case of selection that can be easily detected by the MCOA. No other marker exhibited a similar pattern. A selection signature around the MC1R gene has also been detected using high-density SNP genotyping on dairy cattle breeds (Flori et al. 2009).

The LEIO258 and LEIO228 markers contributed significantly to the multivariate analysis because of their high 
number of alleles. Breeds generally exhibited a higher level of heterozygosity for LEIO258 as compared to microsatellites, which may be expected, because balancing selection rather than directional selection is expected for the MHC locus. However, Ju-Chi and Hsin-Yi showed a rather low heterozygosity, with a quite high frequency of allele 309 in the Ju-Chi, suggesting some selective advantage. Hsin-Yi exhibited the largest difference between observed and expected heterozygosity and a high $F_{\text {Is }}$ value, which was not observed with other markers. A deviation from HardyWeinberg equilibrium for LEIO258 was observed in subsequent generations for this breed (Chang et al. 2010) and suggests some selective pressure on this locus.

\section{Heat tolerance}

A low body weight is generally associated with better heat tolerance and heat evacuation, which is consistent with the positive correlation observed here between mature body weights and panting rate. The higher panting rate of females, in spite of their lower body weight, can be because of a higher level of heat production in mature laying hens than in cockerels, as reported by Macleod et al. (1979). Within the group of heavy breeds (Nagoya, Shek-Ki, HuaTung and Hsin-Yi), Hua-Tung females showed a better heat tolerance, as shown by a lower panting rate. In the light breeds (Ju-Chi and Quemoy), Ju-Chi females showed a better heat tolerance. Thus, differences in adaptation to heat may be observed between local breeds irrespective of their body weight.

\section{Combining phenotypic data and molecular data with a multivariate analysis}

In MCOA, the differentiation of the six breeds according to 24 microsatellite markers matched their geographical distribution. Adding genotypes for MC1R did not greatly change the synthetic position of breeds, but increased the distance between Quemoy and Shek-Ki.

In spite of a significant effect of the breed on performance traits, the PCA analysis using all recorded traits did not discriminate between the six breeds. This could be because of the number of animals per breed, which is low for the study of quantitative variation, but sufficient for the study of molecular variation. Because immune response traits contributed the most to breed differentiation, it is recommended that such traits are included in breed characterization under experimental conditions. For instance, the Ju-Chi and Quemoy clustered together for MC1R and LEIO258 and exhibited similar body weights, but differed significantly for immune traits, with a better antibody response in Quemoy. Further studies should determine whether differences in allelic frequencies at LEIO258, particularly for alleles 309 and 381, could be associated with a different antibody response. It is also possible that trading activity between China and Taiwan through the Quemoy Island increased the exposure of Quemoy chickens to pathogens. Natural selection as well as human selective breeding may have resulted in a stronger antibody response for Quemoy chickens.

Combining performance traits and molecular data with the Hill \& Smith method increased the discrimination between Taiwanese breeds and showed that Hua-Tung, Hsin-Yi and Quemoy did not overlap with each other, but that Ju-Chi did overlap with Quemoy and Hsin-Yi.

Combining the breed history, molecular variability and phenotypic performance makes it possible to classify the six breeds according to three types. The first type corresponds to imported breeds; Shek-Ki and Nagoya were characterized by a heavy body weight and a low heterozygosity for microsatellite markers. They did not have any mtDNA haplotype in common, which is consistent with their quite different histories. Thus, these two breeds illustrate the case of well-established breeds under a long-term conservation programme. Shek-Ki is particularly interesting for further studies regarding the MHC, because it does not share any LEIO258 allele with the Taiwanese breeds. They also showed a much higher heterozygosity level for the LEIO258 marker than for the other markers, supporting the hypothesis of a selective advantage for heterozygous genotypes at the MHC. The second type corresponds to traditional Taiwanese breeds, such as Ju-Chi and the Quemoy, which clustered together for most of the criteria used (MC1R, LEIO258, mtDNA and body weight). From the viewpoint of genetic diversity, it could be proposed that the Quemoy be given conservation priority, as it shows a higher heterozygosity level for microsatellite markers. However, these two breeds differed markedly for immune response traits, and this difference deserves further study. The third type corresponds to more recent breeds such as Hua-Tung and Hsin-Yi, which originated in the last century. Both breeds have been selected for growth and exhibited a higher heterozygosity than the other breeds for microsatellite markers. Hsin-Yi was relatively closer to Ju-Chi and Quemoy for LEIO258 as well as for mtDNA. The mtDNA showed the rather unique origins of Hua-Tung, which could be related to its use as a game breed in the past.

In conclusion, the present study provided a detailed characterization of six local breeds and showed that the conservation programme is successfully maintaining six populations differing in history, molecular polymorphism and performance. The multivariate analysis revealed selection at the MC1R locus, which is supported by the association of MC1R alleles with differences in feather colour. No other locus exhibited such selection in this study. The use of phenotypic data alone could not discriminate breeds efficiently, but the combination of phenotypic and molecular data in a single multivariate analysis provided a better discrimination between breeds than the multivariate analysis with molecular data alone. Regarding performance traits, panting rate revealed interesting differences between 
breeds, irrespective of body weight, and antibody response identified a subset of breeds (Ju-Chi and Quemoy) where the relationships between MHC alleles and immune response should be further studied. Recording immune response traits in association with MHC genotyping could be a useful option for the characterization of local breeds in experimental conditions. The search for selection signatures for disease resistance should be carried out using a much higher density of markers in a set of populations including local breeds from different climatic regions.

\section{Acknowledgements}

This work was supported by grants from the National Science Council of Taiwan (NSC-94-2313-B-005-024 and NSC-97-2313-B-005-002-MY3) and INRA of France. The first author benefited from Institut Français de Taipei (FIT) and NCHU fellowship. Cecile Berthouly was supported by a CIRAD doctoral fellowship and by INRA. Olympe Chazara was supported by a doctoral fellowship from the French ministry of higher education and research.

The authors are grateful to Tatiana Zerjal for comments on this article. The authors gratefully thank the animal caretaker in the experimental farm and many graduate students of NCHU for their assistance in data collection. LEIO258 and mtDNA sequencing was funded by INRA; microsatellite makers genotyping was funded by NSC; MC1R genotyping was supported by NSC, INRA.

\section{References}

Bandelt H.J., Forster P. \& Röhl A. (1999) Median-joining networks for inferring intraspecific phylogenies. Molecular Biology and Evolution 16, 37-48.

Belkhir K., Borsa P., Chikhi L., Raufaste N. \& Bonhomme F. (2004) GENETIX 4.05, Logiciel sous Windows TM pour la génétique des populations. Laboratoire Génome, Populations, Interactions, CNRS UMR 5000. Université de Montpellier II, Montpellier, France.

Berthouly C., Bed'Hom B., Tixier-Boichard M., Chen C.F., Lee Y.P., Laloë D., Legros H., Verrier E. \& Rognon X. (2008) Using molecular markers and multivariate methods to study the genetic diversity of local European and Asian chicken breeds. Animal Genetics 39, 121-9.

Berthouly-Salazar C., Rognon X., Nhu Van T. et al. (2010) Vietnamese chickens: a gate towards Asian genetic diversity. BMC Genetics 11, 53.

Chang C.S., Tixier-Boichard M., Charaza O., Lee Y.P., Chen C.F. \& Bed'hom B. (2010) Monitoring Polymorphism at the Major Histocompatibility Complex Level in Local Chicken Breeds Under a Conservation Program in Taiwan. XIIIth European Poultry Conference, Tours, France.

Chao C.H. \& Lee Y.P. (2001) Relationship between reproductive performance and immunity in Taiwan country chickens. Poultry Science 80, 535-40.

Chen C.F., Berthouly C., Rognon X., Chang K.H., Laloë D., Legros H., Verrier E., Tixier-Boichard M. \& Lee Y.P. (2004) Characterisation of Six Asian Local Chicken Breeds: Performances and Poly- morphism from Microsatellite Markers. 29th International Conference on International Society of Animal Genetics (ISAG), Tokyo, Japan.

Chen C.F., Lee Y.P., Tixier-Boichard M., Bed'hom B., Chang C.M. \& Coville J.L. (2005) Single nucleotide polymorphism in the melanocortin-1 receptor gene of local chickens. Journal of the Chinese Society of Animal Science 34(suppl.), 99.

Chessel D., Dufour A.B. \& Thioulouse J. (2004) The ade4 package-I: one-table methods. R-News 4, 5-10.

Dray S. \& Dufour A.B. (2007) The ade4 package: implementing the duality diagram for ecologists. Journal of Statistical Software 22, http://www.jstatsoft.org/v22/i04/, last accessed on July 15, 2010.

FAO (2007) Status of animal genetic resources. In: The State of the World's Animal Genetic Resources for Food and Agriculture (Ed. by B. Rischkowsky \& D. Pilling), pp. 23-49. Commission on Genetic Resources for Food and Agriculture, Rome.

Flori L., Fritz S., Jaffrézic F., Boussaha M., Gut I., Heath S., Foulley J.L. \& Gautier M. (2009) The genome response to artificial selection: a case study in dairy cattle. PLOS ONE 4, e6595.

Fulton J.E., Juul-Madsen H.R., Ashwell C.M., McCarron A.M., Arthur J.A., O'Sullivan N.P. \& Taylor R.L. Jr (2006) Molecular genotype identification of the Gallus gallus major histocompatibility complex. Immunogenetics 58, 407-21.

Hall T.A. (1999) BioEdit: a user-friendly biological sequence alignment editor and analysis program for Windows 95/98/NT. Nucleic Acids Symposium Series 41, 95-8.

Hill M.O. \& Smith A.J.E. (1976) Principal component analysis of taxonomic data with multi-state discrete characters. Taxon 25, 249-55.

Hillel J., Groenen M.A.M., Tixier-Boichard M. et al. (2003) Biodiversity of 52 chicken populations assessed by microsatellite typing of DNA pools. Genetics Selection Evolution 35, 533-57.

Kerje S., Lind J., Schütz K., Jensen P. \& Andersson L. (2003) Melanocortin 1-receptor (MC1R) mutations are associated with plumage colour in chicken. Animal Genetics 34, 241-8.

Laloë D., Moazami-Goudarzi K. \& Chessel D. (2002) Contribution of individual markers to the analysis of the relationships among breeds by correspondence analysis. 7th World Congress on Genetics Applied to Livestock Production, August 19-23. Montpellier, France.

Laloë D., Jombart T., Dufour A.B. \& Moazami-Goudarzi K. (2007) Consensus genetic structuring and typological value of markers using multiple co-inertia analysis. Genetics Selection Evolution 39, 545-67.

Lamont S.J. (1989) The chicken major histocompatibility complex in disease resistance and poultry breeding. Journal of Dairy Science $72,1328-33$.

Lamont S.J., Pinard-van der Laan M.H., Cahaner A., van der Poel J.J. \& Parmentier H.K. (2003) Selection for disease resistance: direct selection on the immune response. In: Poultry Genetics, Breeding and Biotechnology (Ed. by W.M. Muir \& S.E. Aggrey), pp. 399-418. CABI Press, MA.

Langella O. (1999) Populations 1.2.30 Copyright 1999. CNRS UPR9034, France.

Lee Y.P. (2006) Taiwan country chicken: a slow growth breed for eating quality. In: Symposium, 2006 Scientific Cooperation in Agriculture between Council of Agriculture (Taiwan, R.O.C.) and Institut National de Recherche Agronomique (France) (Ed. by C.W. 
Liao, B.L. Shih, M.L. Lee, A.L. Hsu \& Y.S. Cheng), Technical Bulletin of Livestock Research Institute 103, 121-32.

Ling M.K., Lagerström M.C., Fredriksson R., Okimoto R., Mundy N.I., Takeuchi S. \& Schiöth H.B. (2003) Association of feather colour with constitutively active melanocortin 1 receptors in chicken. European Journal of Biochemistry 270, 1441-9.

Liu Y.P., Wu G.S., Yao Y.G., Miao Y.W., Luikart G., Baig M., BejaPereira A., Ding Z.L., Palanichamy M.G. \& Zhang Y.P. (2006) Multiple maternal origins of chickens: out of the Asian jungles. Molecular Phylogenetics and Evolution 38, 12-9.

Macleod M.G., Tullett S.G. \& Jewitt T.R. (1979) Effects of food intake regulation on the energy metabolism of hens and cockerels of a layer strain. British Poultry Science 20, 521-31.

Mundy N.I. (2005) A window on the genetics of evolution: MC1R and plumage colouration in birds. Proceedings of the Royal Society Biological Sciences 272, 1633-40.

Nei M., Tajima F. \& Tateno Y. (1983) Accuracy of estimated phylogenetic trees from molecular data. Journal of Molecular Evolution 19, 153-70.

R Core Development Team (2006) R: A Language and Environment for Statistical Computing. R Foundation for Statistical Computing, Vienna, Austria. ISBN 3-900051-07-0, http://www.R-project. org.

SAS Institute. (2008) SAS/STAT User's Guide. Release 9.1.3 Edition. SAS Institute Inc., Cary, NC.

Smyth J.R. (1996) Genetics of plumage, skin and pigmentation in chickens. In: Poultry Breeding and Genetics (Ed. by R.D. Crawford), pp. 109-67. Elsevier Science, New York.

Staden R., Beal K.F. \& Bonfield J.K. (1998) The Staden package. Methods in Molecular Biology 132, 115-30.

Takeuchi S., Suzuki H., Yabuuchi M. \& Takahashi S. (1996) A possible involvement of melanocortin 1-receptor in regulating feather color pigmentation in the chicken. Biochimica et Biophysica Acta 1308, 164-8.
Weaver W.D. Jr (2002) Poultry housing. In: Commercial Chicken Meat and Egg Production, 5th edition (Ed. by D.D. Bell \& W.D. Weaver Jr), pp. 101-11. Kluwer Academic Publishers Press, MA. Yang C.Y., Shieh H.K., Lin Y.L. \& Chang P.C. (1999) Newcastle disease virus isolated from recent outbreaks in Taiwan phylogenetically related to viruses (genotype VII) from recent outbreaks in Western Europe. Avian Diseases 43, 125-30.

\section{Supporting information}

Additional supporting information may be found in the online version of this article.

Figure S1 A (upon)- Neighbor-joining tree analysis of the MC1R gene for the six local breeds. B (down)- Neighborjoining tree of the LEI0258 marker for the six local breeds. Table S1 General characteristics of the 24 microsatellite markers for the six breeds.

Table S2 Description of the primers used in this study. PCR regions and amplification lengths, names and primer sequences are shown.

Table S3 The semi-matrix of Fst in six local breeds.

Table S4 MC1R genotypes, animal number and percentage in each breed.

As a service to our authors and readers, this journal provides supporting information supplied by the authors. Such materials are peer-reviewed and may be re-organized for online delivery, but are not copy-edited or typeset. Technical support issues arising from supporting information (other than missing files) should be addressed to the authors. 